\title{
THE INTELLIGENT QUADRIFILAR HELIX: A COMPACT MIMO ANTENNA FOR IEEE 802.11n
}

\author{
T.W.C. Brown, S.R. Saunders \\ Centre for Communication Systems Research, University of Surrey, Guildford, Surrey, GU2 7XH, UK, \\ www.ee.surrey.ac.uk/CCSR, t.brownesurrey.ac.uk, simonesimonsaunders.com
}

Keywords: MIMO, Intelligent Quadrifilar Helix Antenna, Measurements

\begin{abstract}
A compact MIMO antenna solution, the intelligent quadrifilar helix antenna for the IEEE 802.11n standard is proposed. Analysis has been made with regard to design considerations and preliminary narrowband measurements have been carried out to evaluate its capacity potential in comparison to conventional spatially separated linear antennas. The results indicate that there is comparable capacity potential with nearly $70 \%$ reduction in size.
\end{abstract}

\section{Introduction}

The recently-agreed IEEE 802.11n standard for Wi-Fi creates a strong incentive for consumer-friendly MIMO antennas in both client devices and access points, delivering higher data rates and increased ranges for a wide range of applications. Pre-standard implementations of IEEE 802.11n and many publications have used multiple spatially separated linearly polarised antennas, but these are neither compact nor attractive in a consumer deployment and also add to implementation cost. Some publications have therefore considered compact alternatives [2], [5]-[10] though there are relatively few examples of such solutions as fully deployed antennas. This paper proposes the intelligent quadrifilar helix antenna (IQHA) [8] illustrated in Figure 1, for both the access point and for selected client device applications. This antenna operates with a mixture of spatial, polarisation and angular diversity modes, delivering measured and simulated diversity gains of up to $13 \mathrm{~dB}$ at $1 \%$ fading probability [3] via four branches.

Initial analysis of the IQHA has shown its potential as a MIMO antenna, with comparable performance to halfwavelength spaced monopoles [4] in a compact form. However, these results are based on analysing the antenna pattern correlation between the four elements in the helix from which an approximate idea of the MIMO data throughput (or capacity) can be determined. This analysis, however, is based on crude channel assumptions that do not always hold in a typical radio environment. Therefore to gain a more realistic evaluation of the MIMO performance, this paper presents some preliminary measurements taken in a narrowband fading environment with a channel sounder.

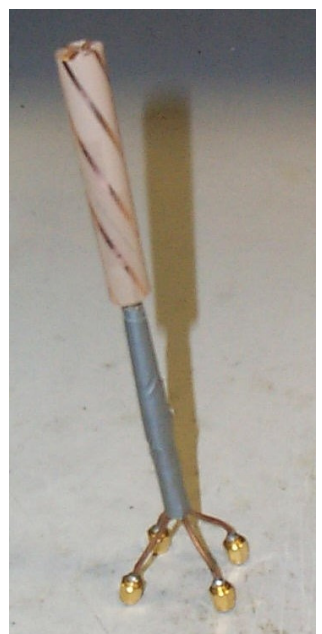

Figure 1 - Example intelligent quadrifilar helix antenna (IQHA)

\section{Antenna Design Parameters}

There are four main factors to optimal design of an IQHA for IEEE $802.11 \mathrm{n}$ being the compactness of size, low correlation between the four elements, high efficiency and bandwidth. The properties of the IQHA will be affected by different dimensions [1] as illustrated in Figure 2 with just one helix for clarity. The same dimensions apply to an IQHA. Therefore the following factors have to be considered:

- $\quad$ Diameter, $d$, and antenna length $L$ (which will in turn determine the wire length of each helix element). Both of these dimensions need to be practical, which usually requires $L$ and $d$ being small. However, if either is set too low, this will reduce the number of possible turns, $N$, and element length, which will result in high branch correlation. Furthermore the element length, $l_{\text {elem }}$ needs to be a minimum value (usually three quarters of a wavelength) so that the antenna has suitable efficiency and an impedance match.

- The spacing between turns, $s$, and their resultant pitch angle, $\alpha$. This should not be too close or the mutual coupling between elements will increase, which will in turn degrade the efficiency.

- The IQHA's weakness is that it can be limited in bandwidth, though it can be widened to around $15 \%$ 
through wider wire diameter (which would be acceptable for IEEE 802.11n that uses carriers much narrower in bandwidth).

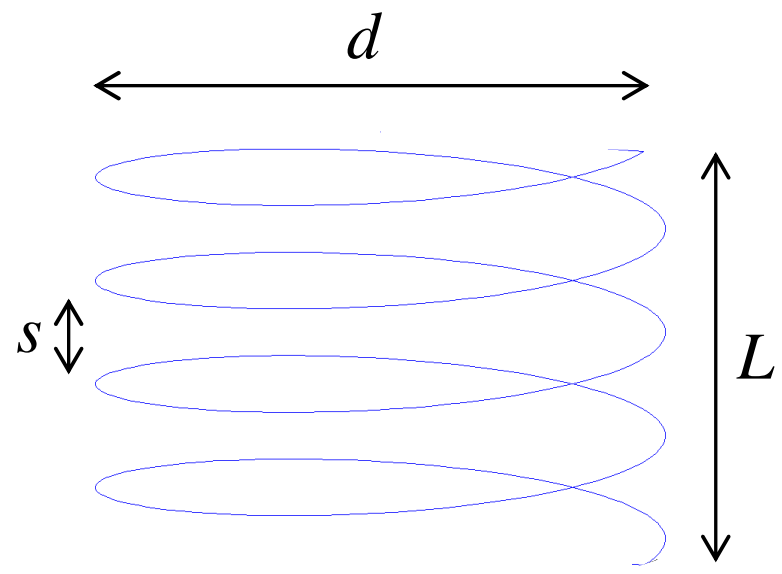

\section{Number of turns, $N \quad \alpha=\frac{s}{\pi d}$}

Figure 2 - Illustration of the dimensions of a helix antenna

A brief analysis is made in Table 1 of the impact of antenna design on branch correlation and mean effective gain (MEG) by applying different antenna dimensions at $2.4 \mathrm{GHz}$ simulated in numerical electromagnetic code (NEC). The environment conditions of uniform azimuth and Gaussian elevation angle of arrival along with a cross polar ratio of $6 \mathrm{~dB}$ are applied as in [3]. In this case, the element length, $l_{\text {elem }}$ is fixed to 0.75 wavelengths (i.e. $93.75 \mathrm{~mm}$ ) and $N$ is fixed to 0.75 . By using the theory presented in [1], $L$ is related to $d$ by the following equation:

$$
L=N \sqrt{\left(\frac{l_{\text {elem }}}{N}-d\right)^{2}-\pi d^{2}}
$$

\begin{tabular}{|c|c|c|c|c|}
\hline $\boldsymbol{d}(\mathbf{m m})$ & $\boldsymbol{L}(\mathbf{m m})$ & $\left|\boldsymbol{\rho}_{\mathbf{1 2}}\right|^{\mathbf{2}}$ & $\left|\boldsymbol{\rho}_{\mathbf{1 3}}\right|^{\mathbf{2}}$ & $\begin{array}{c}\text { MEG } \\
(\mathbf{d B i})\end{array}$ \\
\hline 2.5 & 91.81 & 0.55 & 0.53 & -4.96 \\
\hline 5 & 89.75 & 0.46 & 0.46 & -5.02 \\
\hline 10 & 85.21 & 0.28 & 0.25 & -5.92 \\
\hline 15 & 80.05 & 0.01 & 0.01 & -3.89 \\
\hline 20 & 91.81 & 0.03 & 0.08 & -4.46 \\
\hline
\end{tabular}

Table 1 - Comparison of the branch correlation and mean effective gain (MEG) from different IQHA diameters and subsequent lengths at $2.4 \mathrm{GHz}$

By taking cases of $d$ in Table 1 and then the subsequent $L$ and branch correlation (where $\rho_{12}$ is equal to $\rho_{14}$ ), it can be seen that correlation increases with $d$, though there are cases where it is not optimum and also MEG will increase and decrease at various points. A practical size with good de-correlation and higher MEG occurs between $10 \mathrm{~mm}$ and $15 \mathrm{~mm}$. Likewise a practical IQHA within this region has been applied to the measurement setup in the next section.

\section{Measurement Setup}

The frequency of operation chosen, due to the scope of the channel sounder used and the available spectrum was $2.4 \mathrm{GHz}$, which is close to frequencies used in IEEE 802.11n. The sampling rate used was $62 \mathrm{~Hz}$, over five times the maximum Doppler shift. There was need for such high sampling not just to avoid aliasing but also due to the fact that there were interferences present from wireless local area networks (WLANs) in the surrounding area that caused occasional disruption to the measurements. In light of this, the data was post processed to remove these intermittent interferences and then replace them with interpolated data. High sampling therefore enabled negligible effect on the resultant impulse responses that were post processed into narrowband fading channels.

The IQHA under test is shown in Figure 3 (a) with diameter $13 \mathrm{~mm}$ and height $60 \mathrm{~mm}$. There are 0.75 turns, with the element length approximately 0.75 wavelengths though shortened a few percent in order to attain impedance matching. Ferrites are also applied to alleviate current imbalance and cabling effects.

The vertically orientated IQHA was measured along the same test run as a set of four horizontally spaced vertical dipoles as shown in Figure 3 (b). The spacing was half a wavelength so as to achieve good theoretical de-correlation and also each dipole was fed with a balun to avoid current imbalance.

By considering the maximum dimensions of both antenna arrangements, the IQHA has $60 \mathrm{~mm}$ height and the total width of the dipoles is around $190 \mathrm{~mm}$. Therefore there is nearly $70 \%$ reduction in size with an IQHA compared to conventional spatial multiplexing.

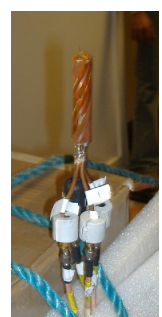

(a)

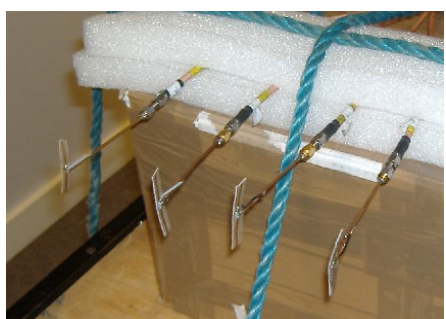

(b)
Figure 3 - Picture of the (a) four half wavelength dipoles and (b) IQHA under test

As illustrated in Figure 4 and Figure 5, the measurement was carried out in a non line of sight (NLOS) multipath rich corridor environment, where the fixed transmit antenna was based upon four directional patch antenna elements. The IQHA and the monopoles were then placed at the mobile, attached to the same cables. All four cables were assumed to have equal attenuation for calibration purposes. Therefore the IQHA and the four dipoles were measured on the front ends 
of the same cables to enable a fair test. It is assumed that the two antennas are taken through the same environment and tests were repeated to ensure repeatability of the data collected. During the measurement run, the trolley was moved along the path at walking pace.

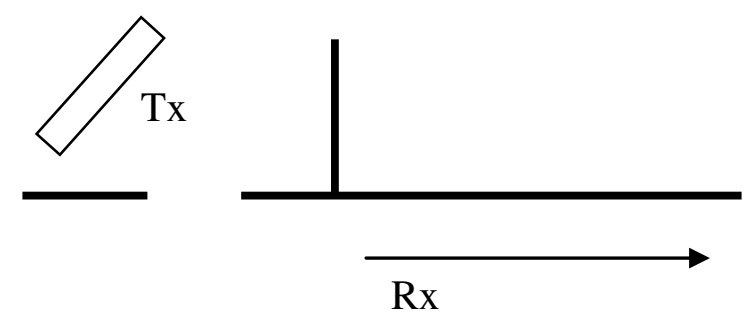

Figure 4 - Diagram of the measurement campaign test run

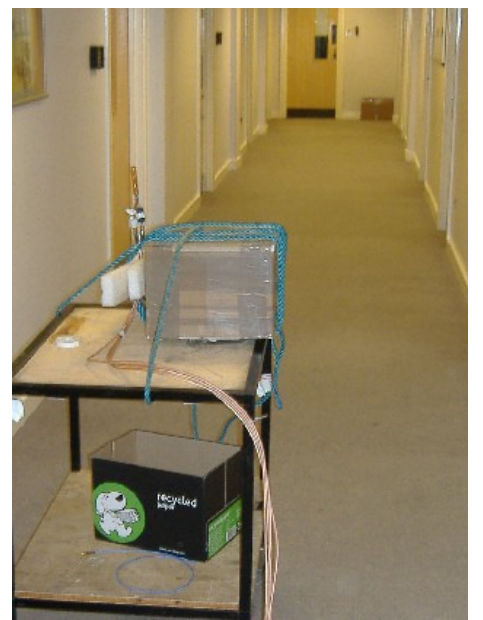

Figure 5 - Picture of the measurement campaign test run

\section{Results of Preliminary Measurements}

To begin with, the data from both measurements was analysed to gain a fair comparison between the two antennas. To analyse purely the difference in scattering (which will be changed with different antenna patterns) the path loss was first normalised out for the spatial dipoles, so only the fading of the 16 paths is analysed. Therefore to normalise the channel for the spatial dipoles, $\mathbf{H}_{\text {spatial }}$, the branch with the maximum path mean level received at the mobile using the first branch at the transmit end, $\max \left(\left|h_{\text {spatial } 1 n}\right|\right)$, where $n$ ranges from 1 to 4 , was used to normalise the channel to $\mathbf{H}_{\text {spatial }}$ so that:

$$
\mathbf{H}_{\text {spatial }}{ }^{\prime}=\frac{\mathbf{H}_{\text {spatial }}}{\max \left(\left|h_{\text {spatial } 1 n}\right|\right)}
$$

Therefore, to allow a fair comparison to the spatial case, the channel for the IQHA, $\mathbf{H}_{I Q H A}$, is normalised also by $\max \left(\left|h_{\text {spatial1 } n}\right|\right)$ because if there is any reduction in antenna efficiency within the IQHA, this will then be included in the comparison because it will create resultant loss in signal to noise ratio (SNR). Branch power ratio at the mobile should there be any is also accounted for at the mobile.

$$
\mathbf{H}_{I Q H A}{ }^{\prime}=\frac{\mathbf{H}_{I Q H A}}{\max \left(\left|h_{\text {spatial } 1 n}\right|\right)}
$$

By assuming that both antennas are experiencing the same radio environment when measured, there is now a suitable scenario where a direct comparison can be made regarding their ergodic capacity due to scattering. To start with, the correlation of the antennas is analysed in Table 2, where antenna branches 1, 2, 3 and 4 are compared with branch 1 in both cases. The results would be repeated if comparing relative to any other branch. As can be seen, the spatial dipoles all have low correlation, as would be expected with half wavelength spacing. For the IQHA, there is higher correlation for $\left|\rho_{13}\right|^{2}$ though it is still below 0.7 , which as far as diversity is concerned is still low enough correlation.

\begin{tabular}{|l|l|l|l|l|}
\hline & $\left|\rho_{11}\right|^{2}$ & $\left|\rho_{12}\right|^{2}$ & $\left|\rho_{13}\right|^{2}$ & $\left|\rho_{14}\right|^{2}$ \\
\hline Dipoles & 1 & 0.08 & 0.01 & 0.12 \\
\hline IQHA & 1 & 0.08 & 0.61 & 0.16 \\
\hline
\end{tabular}

Table 2 - Comparison of the correlation between branches for four dipoles and an IQHA

In order to analyse their scattering, it is necessary to undertake an eigenvalue analysis of both cases, so that the resultant channel is decomposed by eigenvalue decomposition (EVD) such that the eigenvalues, $\mathbf{S}$ and eigenvectors, $\mathbf{V}$, are related by:

$$
\mathbf{H H}^{H}=\mathbf{V S V}^{H}
$$

The matrix $\mathbf{S}$ is a diagonal matrix with four eigenvalues, $s_{1}$, $s_{2}, \quad s_{3}$ and $s_{4}$. Their cumulative distribution from measurements undertaken is plotted in Figure 6, where there is shown to be good consistency for the distributions of the first and second eigenvalues but for the third and fourth there is a shift. However, in the case of the IQHA, there is an increase in the third eigenvalue, which results in three stronger sub-channels, where the fourth is often of little significance. 


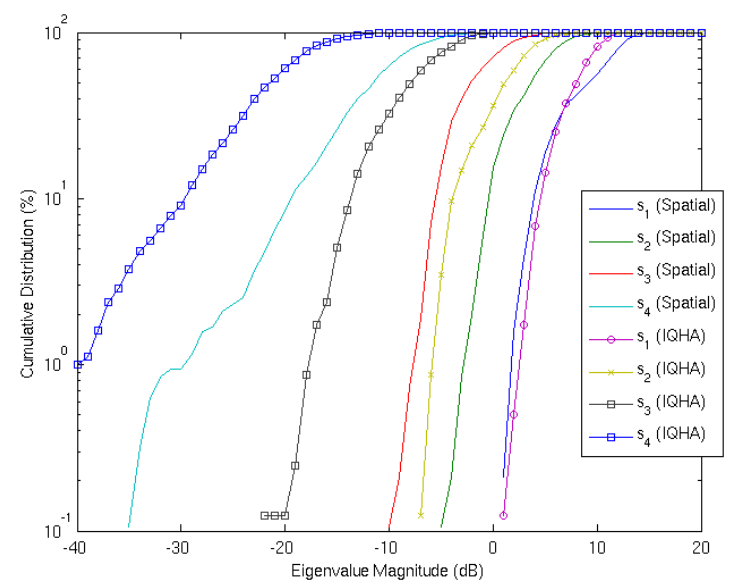

Figure 6 - Comparison of the eigenvalue distributions between an four half wavelength spaced dipoles and an IQHA

In both cases, the capacity can be calculated based on the resolved eigenvalues, such that the Shannon capacity, $C$ in bits/s/Hz is resolved by [4]:

$$
C=\log _{2}|\mathbf{I}+\mathrm{SNR} . \mathbf{S}|
$$

where I is the identity matrix and SNR is the signal to noise ratio. The cumulative distribution of the capacity when SNR is $10 \mathrm{~dB}$ is plotted in Figure 7, where it can be seen that there is a slight drop in capacity when comparing the IQHA against the spaced dipoles. This will be partly due to the higher correlations seen in the IQHA but more so the small loss in gains which is evident when comparing the $3^{\text {rd }}$ and $4^{\text {th }}$ eigenvalues in Figure 6. Such loss will cost an increase in SNR of $2-3 \mathrm{~dB}$ to attain comparable capacity. However, the reduction in size is as much as $70 \%$ in the case of the IQHA, which is more realistic and practical. Further work will investigate different uses, applications and better optimised designs to achieve maximum capacity potential. This paper provides results in the initial stages.

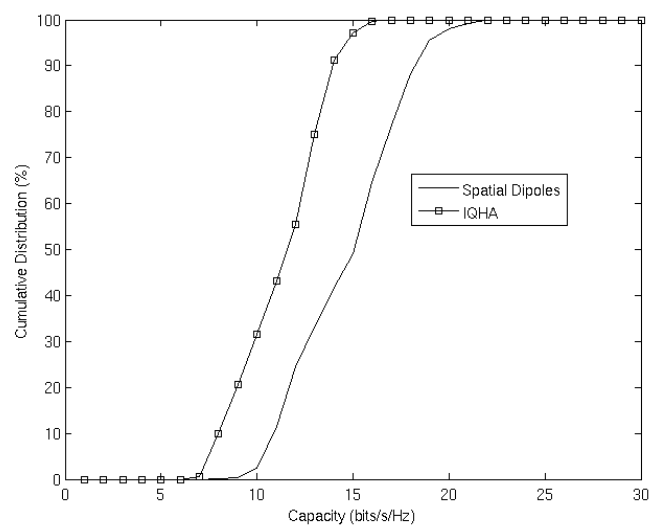

Figure 7 - Comparison of the 4x4 MIMO capacity with four half wavelength spaced dipoles and an IQHA $(\mathrm{SNR}=10 \mathrm{~dB})$

\section{Conclusions}

Initial analysis of the IQHA at $2.4 \mathrm{GHz}$ has been presented both in terms of the antenna design and also preliminary results carried out in a small scale non line of sight indoor measurement campaign. Preliminary indications show that the antenna does have capacity potential comparable with that of four half wavelength spaced dipoles, giving a size reduction of up to $70 \%$, which is attractive for developing compact MIMO systems for IEEE 802.11n applications.

\section{References}

[1] A-A. Agius, "Antennas for Handheld Satellite Personal Communicators", PhD Thesis, University of Surrey, UK, (1999).

[2] R. Bains, R. Müller, "Appropriate Antenna Patterns for a Compact MIMO Receiver", International Zurich Seminar on Communications, (February 2006).

[3] T. W. C. Brown, K. C. D. Chew, S. R. Saunders, "Analysis of the Diversity Potential of an Intelligent Quadrifilar Helix Antenna", IEE 12th International Conference of Antennas and Propagation (ICAP), no. 491 vol. 1, pp194-197, (April 2003).

[4] K. C. D. Chew, L. Morfis, D. Mavrakis, S. Stavrou, "Quadrifilar helix antenna for MIMO system", Antennas and Wireless Propagation Letters, vol. 3 , pp197-199, (2004).

[5] A. Forenza, T. Heath, "Benefit of Pattern Diversity via Two-Element Array of Circular Patch Antennas in Indoor Clustered MIMO Channels", IEEE Transactions on Communications, vol. 54, no. 5, (May 2006).

[6] B. N. Getu, J. B. Andersen, "The MIMO Cube - A Compact MIMO Antenna”, IEEE Transactions on Wireless Communications, vol. 4, no. 3, pp11361141, (May 2005).

[7] A. S. Konanur, K. Gosalia, S. H. Krishnamurthy, B. Hughes, G. Lazzi, "Increasing Wireless Channel Capacity Through MIMO Systems Employing CoLocated Antennas", IEEE Transactions on Microwave Theory and Techniques, vol. 53, no. 6, (June 2005).

[8] S. M. Leach, A. A. Agius, S. R. Saunders, "Intelligent quadrifilar helix antenna", IEE Microwaves, Antennas and Propagation, vol. 147, no. 3, pp219-223, (June 2000).

[9] M. Stoytchev, H. Safar, A. L. Moustakas, S. Simon, "Compact antenna arrays for MIMO applications", IEEE Antennas and Propagation Society International Symposium, vol. 3, pp708-711, (2001).

[10] C. Waldschmidt, W. Wiesbeck, "Compact WideBand Multimode Antennas for MIMO and Diversity", IEEE Transactions on Antennas and Propagation, vol. 52, no. 8, pp1963-1969, (August 2004). 